\title{
M-learning: Estrategia para la promoción del aprendizaje electrónico móvil en instituciones de educación superior
}

\section{M-learning: strategy for the promotion of mobile e-learning in higher education institutions}

\author{
Raysa Chiquinquirá Chirino-García \\ rchirino@urbe.edu.ve \\ Universidad Privada Dr. Rafael Belloso Chacín, Maracaibo \\ Venezuela \\ https://orcid.org/0000-0002-0336-2870 \\ Janeth Hernández-Corona \\ jchernandez3@urbe.edu \\ Universidad Privada Dr. Rafael Belloso Chacín, Maracaibo \\ Venezuela \\ https://orcid.org/0000-0002-2274-687X
}

Recepción: 1 Noviembre 2019

Revisado: 15 de diciembre 2019

Aprobación: 15 febrero 2019

Publicación: 25 de febrero 2020

\section{RESUMEN}

El artículo de revisión se orientó en analizar el M-learning como estrategia para la promoción del aprendizaje electrónico móvil en instituciones de educación superior en Venezuela. El abordaje se gestó desde la perspectiva analítica, desarrollándose un proceso reflexivo por parte de las investigadoras empleándose un tipo de investigación documental. Se deja en evidencia que el M-Learning de ninguna manera representa un sustituto de la formación tradicional pues ambas pueden coexistir perfectamente en función de las necesidades, además dado el vertiginoso auge de las nuevas tecnologías, se puede afirmar que los estudiantes conocen bien los diferentes medios informáticos pero los docentes (profesores, tutores y facilitadores, entre otros) son los que manejan la tecnología cognitiva, además que, su misión es enseñarles cómo optimizar y usar dichos dispositivos o instrumentos móviles pero con fines académicos; de ahí el reto al que deben enfrentarse. 
Palabras clave: Enseñanza asistida por ordenador, informática educativa, tecnología educacional, enseñanza multimedia (Palabras tomadas del Tesauro UNESCO).

\begin{abstract}
The review article was focused on analyzing M-learning as a strategy for promoting mobile electronic learning in higher education institutions in Venezuela. The approach was created from an analytical perspective, developing a reflective process by the researchers and using a type of documentary research. It is evident that M-Learning by no means represents a substitute for traditional training since both can coexist perfectly depending on the needs; in addition, given the dizzying rise of new technologies, it can be said that students are well aware of the different computer media but educators (teachers, tutors and facilitators, among others) are the ones who handle cognitive technology, considering that its mission is to teach them how to optimize and use such mobile devices or instruments but for academic purposes; hence the challenge they must face.
\end{abstract}

Keywords: Computer assisted instruction, computer uses in education, educational technology, multimedia instruction (Words taken from UNESCO Thesaurus).

\title{
INTRODUCCIÓN
}

El Internet asociado a la incorporación de nuevos medios electrónicos ha aparejado un cambio sustancial en todas las esferas sociales, al modificar radicalmente muchas prácticas y actividades cotidianas. En efecto, se ha generado la era móvil digital, la cual impone cambios en el entorno empresarial, social y educativo, dado el dinamismo de la tecnología de la Información. Por consiguiente, resulta perentorio que la sociedad ante esas evidentes reformas ejerza presión al sector educativo para que asuma parte activa en dicho proceso (Perozo Martín \& Chirinos Martínez, 2019).

Por otro lado, esto apareja una ruptura en los paradigmas tradicionales del sector educativo pues la revolución tecnológica otorga una apertura a otras estructuras que inciden en la educación; estando a la vanguardia en las nacientes modalidades de negocios, propiciándose la satisfacción del mercado empresarial. En este contexto, los 
nuevos escenarios estarán jerarquizados por la coexistencia informativa, revistiendo importancia, pues las fuentes de información constituyen un elemento esencial para la toma de decisiones; por tanto, las nuevas tecnologías serán fuentes capaces de brindar el soporte necesario para las instituciones de educación superior que coadyuve con el desarrollo de actitudes - aptitudes en los individuos (Anderson, \& Andara, 2017).

Por otro lado, el uso diverso otorgado actualmente a los ambientes virtuales de aprendizaje han conducido la integración cada día en mayor cantidad de estudiantes, permitiendo además dar solución a la problemática del espacio físico, aunado a la administración del tiempo, puesto que se reducen los mismos además de dar respuesta al mundo globalizado en materia de educación pues se genera praxis de un currículo flexible, colaborativo, cooperativo (Peche Cruz, \& Giraldo Supo, 2019).

En este sentido, las nuevas maneras de transferencia de conocimientos conjuntamente con el uso generalizado de las TIC, además de materiales que propician el aprendizaje fomentan la asimilación del conocimiento. En otras palabras, la educación mediante el uso de ambientes virtuales en la práctica despeja la inquietud de cómo el proceso de enseñanza-aprendizaje se mejora investigando (Mero Chávez, Fernando Pazmiño \& San Andrés, 2019).

De ahí que, existe un mejor aprovechamiento del tiempo dedicado al desarrollo de algunas competencias del individuo, para así poder aplicar esos momentos a la profundización y estudio de conceptos, procesos que impliquen la solución de determinados problemas (Aldana Zavala, 2019). En virtud de lo planteado anteriormente, esta investigación se orientó en analizar el M-learning como estrategia para la promoción del aprendizaje electrónico móvil en instituciones de educación superior en Venezuela. 
EPISTEME KOINONIA

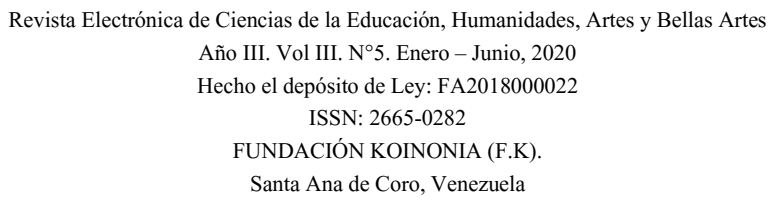

Raysa Chiquinquirá Chirino-García; Janeth Hernández-Corona

\section{MÉTODO}

El abordaje se gestó desde la perspectiva analítica, desarrollándose un proceso reflexivo por parte de las investigadoras (Aldana Zavala, 2019), empleándose un tipo de investigación documental. En este sentido, (Palella y Martins, 2012) la definen como aquella que se concreta exclusivamente en la recopilación de información en diversas fuentes documentales escritas u orales, teniéndose como población de estudio, textos, artículos científicos, documentos oficiales. Complementándose en un estudio descriptivo (Arias, 2012), indica que esta consiste en la caracterización de un hecho, fenómeno, individuo o grupo, abordándose desde un diseño bibliográfico, aplicándose la técnica de análisis de contenido para estructurar los diversos análisis categoriales.

\section{ANÁLISIS Y DISCUSIÓN DE LOS RESULTADOS}

\section{Aprendizaje electrónico}

Para Cabal, Martínez y otros (2010), el aprendizaje electrónico conocido también como e-learning por el anglicismo Electronic Learning se puede definir como el proceso de enseñanza-aprendizaje que se desarrolla mediante el uso de redes, internet, así como a través de la utilización de medios electrónicos. Por otro lado, Xiao \& Benbasat (2007), comentan que éste puede ser definido como una modalidad de aprendizaje dentro de la educación a distancia en la que se utilizan las redes de datos como medios (Internet, Intranets, entre otros), las herramientas o aplicaciones hipertextuales como soporte (Correo electrónico, web, Chat, entre otros) así como los contenidos y/o unidades de aprendizaje en línea.

Desde otra óptica, la (Organización de las Naciones Unidas para la Educación, la Ciencia y la Cultura UNESCO, 2019) indica que es un concepto más relacionado con lo semi presencial, también es llamado b-learning'(blendedlearning). Del mismo modo, advierte que el aprendizaje electrónico es la piedra angular para construir sociedades 


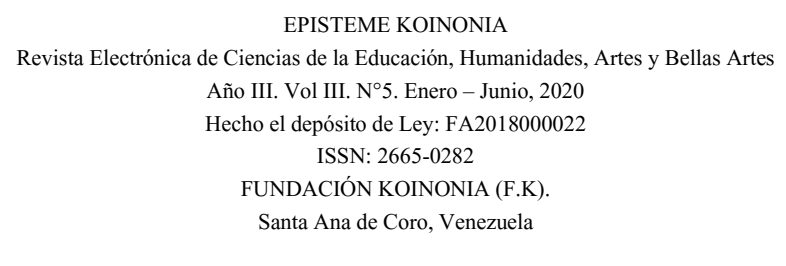

Raysa Chiquinquirá Chirino-García; Janeth Hernández-Corona

integradoras del conocimiento. En este sentido, la UNESCO, en su mandato singular de promover la libre circulación de las ideas y el conocimiento, ha desempeñado una función esencial en la Cumbre Mundial sobre la Sociedad de la Información pues su contribución incorporó los aspectos éticos, jurídicos y socioculturales de la sociedad de la información además de contribuir al aprovechamiento de las oportunidades que ofrecen las TIC, al situar al ser humano en el centro del enfoque.

Sobre la base de las ideas planteadas, se puede asentir que el aprendizaje electrónico infiere aquella enseñanza por medio de equipos electrónicos, tales como computadora, correo electrónico, la red www y la tecnología del CD-ROM; por lo que de esta forma ofrece una nueva posibilidad de capacitación puesto que la sociedad globalizada requiere aprender rápidamente nuevas técnicas además de asimilar otro tipo información, para competir eficazmente.

\section{Ventajas del aprendizaje electrónico}

En este aparte se efectúa de algunas ventajas que ofrece la formación en línea o educación a distancia. En este sentido, las instituciones de educación superior deberán evaluar las formas que ha adoptado la llamada educación a distancia, al desarrollarse y masificarse las intranets y redes de área amplia dado que los métodos de aprendizaje electrónico (e-learning) son tan eficaces para la enseñanza o aún más, que las aulas tradicionales.

De ahí que, éstos pueden proporcionar oportunidades de aprendizaje a los individuos que de otra manera no tendrían acceso a nuevos conocimientos; adicionalmente, las combinaciones de aprendizaje en aulas y aprendizaje electrónico podrían constituir a una mejor estrategia en las instituciones de educación. En esta instancia, Velazco Flórez, Abuchar Porras, Castilla, \& Rivera, (2017), señalan como beneficios del aprendizaje electrónico, los siguientes: 
a. Ahorro. El e-learning supone una reducción de costos y gastos significativos si se compara con el aprendizaje presencial pues con el aprendizaje en línea se logran ahorros sustanciales de traslado, material didáctico, entre otros.

b. Rapidez y agilidad. Se erige sobre la enorme ventaja de ser un aprendizaje donde predomina la inmediatez a la hora de obtener la información necesaria para cualquier tipo de aprendizaje. La información se consigue al momento, de forma ágil y muy accesible.

c. Acceso just-in-time. La formación a través del e-learning se lleva a cabo en el momento en que se necesita. En este sentido, es el usuario que básicamente controla el tiempo de tu formación en línea.

d. Acceso just-for-me. Además de poder realizar un aprendizaje en el momento en que se desee, éste facilita un tipo de enseñanza-aprendizaje mucho más individualizado y personalizado en función de las características tanto pedagógicas como tecnológicas.

e. Flexibilidad horaria. Otra clara ventaja del e-learning en contraposición al aprendizaje presencial es que el usuario puede gestionar tu propio ritmo de aprendizaje. Asimismo, puede hacerlo a cualquier hora y en cualquier lugar, siempre y cuando tenga a su disposición una conexión a internet o un dispositivo electrónico. Por otro lado, mediante el uso del e-learning se consigue romper la siempre difícil barrera temporal.

f. Material electrónico. Este sustituye el formato en papel por un formato electrónico. Principalmente se trata de un material audiovisual como, por ejemplo, video tutoriales o presentaciones, entre otros.

g. Barrera espacial. Con el e-learning las barreras espaciales desaparecen pues es posible realizar cualquier tipo de formación en línea sin importar la ubicación geográfica del usuario. Es lo que se entiende como deslocalización espacial.

h. Actualización. En el e-learning los contenidos son en línea; ello es, gozan de la ventaja de estar alojados en espacios donde se pueden revisar y actualizar constantemente. En este sentido, la ventaja no es una actualización unidireccional, sino que pueden realizarla tanto docentes como estudiantes.

i. Multidispositivo. El e-learning permite el aprendizaje de cualquier tipo de enseñanza a partir de una amplia gama de dispositivos. Con el e-learning es posible acceder a cualquier formación a partir de un smartphone, una tablet o un ordenador.

j. Conciliación. Al ser un tipo de enseñanza deslocalizada, el e-learning permite algo fundamental en la sociedad actual: la conciliación entre la vida familiar y la 
vida laboral. El e-learning hace posible que el aprendizaje y la vida familiar sean compatibles, precisamente porque permite la flexibilidad horaria aludida anteriormente.

k. Tutorización. Este es un elemento fundamental en el e-learning. El hecho que los alumnos no puedan tener un contacto directo con el docente que imparte una determinada enseñanza, no es impedimento para que se realice una supervisión del progreso o avance del estudiante mediante la tutorización. Existen varias formas por las cuales se puede tener contacto fluido con el docente/facilitador a través de correo electrónico o videoconferencia, entre otros.

I. Aprendizaje colaborativo. Aunque en el e-learning el aprendizaje es principalmente individual, ello no obsta que cierto tipo de enseñanzas se centren en lo que se denomina el aprendizaje colaborativo. Se trata de un aprendizaje virtual, pero en el que a través de una determinada plataforma distintas personas pueden realizar actividades, debates, lluvias de ideas para ponerlas en común. Un claro ejemplo de plataforma colaborativa serían los hangouts de Google.

m. Plataforma virtual. Además de una conexión a internet, así como un dispositivo electrónico, en el e-learning suele ser común el uso de algún tipo de plataforma donde se concentra toda la información relativa a una enseñanza. En educación una de las plataformas más consolidadas es la plataforma Moodle.

n. Gestión de la información. La gestión de la información mediante el e-learning es claramente distinta a la que se maneja en una formación presencial. En el elearning el estudiante parte con un amplio volumen de información a la que tiene acceso de forma libre.

o. Titulación. Cada vez más son las enseñanzas realizadas mediante el e-learning que tienen una titulación homologada al igual que cualquier formación presencial. Este aspecto sin duda ha hecho que mucha gente haya optado por este tipo de aprendizaje electrónico.

Volviendo la mirada hacia los contenidos planteados anteriormente, es preciso exhortar a las instituciones de educación superior sobre la promoción que han de realizar sobre el comercio y aprendizaje electrónico pues estas ofrecen los medios para apoyar la capacitación así como la formación que coadyuve a la transferencia del conocimiento puesto que estas novedosas modalidades en la educación rompen con el aislamiento espacial y temporal lo cual se traduce en iniciativas de mejoras para la sociedad. 


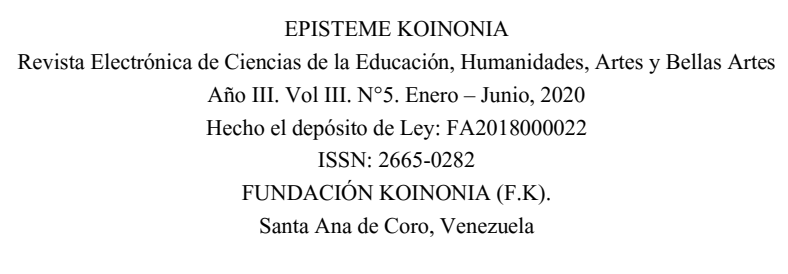

Raysa Chiquinquirá Chirino-García; Janeth Hernández-Corona

\section{Aprendizaje electrónico móvil}

Para efectuar el abordaje de este punto, resulta necesario puntualizar algunos términos con el fin de evitar confusiones por la utilización de terminologías análogas. De ahí que, el aprendizaje móvil también conocido como M-learning, esta referido a los ambientes de aprendizaje basados en la tecnología móvil, destinados a mejorar e impulsar los procesos de enseñanza y aprendizaje. De esta manera se puede advertir que el aprendizaje móvil está relacionado con la educación fuera del aula; ello es, educación a distancia.

En este sentido, Hernández \& Morales (2010), sostienen que el término distancia implica un cambio geográfico entre donde residen los contenidos; es decir, el lugar en el que se toman, manteniendo siempre una conexión física entre ellos. En cambio, en el M-Learning el término distancia implica que la recuperación o el acceso al contenido puede hacerse en movimiento, sin importar el lugar y obteniendo un mayor provecho del tiempo disponible.

Desde otra perspectiva Kinshuk (2009) advierte que es una tendencia producto de la proliferación de los sistemas de comunicación actuales. Por su parte, Quinn (2010) lo concibe como la confluencia entre computación móvil y aprendizaje electrónico, pero con accesibilidad a los recursos requeridos, así como el soporte de aprendizaje efectivo, según Brazuelo Grund \& Gallego Gil (2011), indican que el M-Learning se presenta como un apoyo a los procesos educativos de carácter móvil, los cuales requieren de alta interactividad en el desarrollo del aprendizaje, con integración de contenidos y ubicuidad en actividades cognitivas.

Ahora bien, en estos tiempos, se infiere lo inherente a la tecnología cognitiva, al respecto Cavaller (2016), comenta que ésta es la clave para el crecimiento; de ahí que, los autores la conciben como "La línea que separa los resultados de la analítica automatizada y la tecnología cognitiva es relativamente delgada", puesto que muchas 


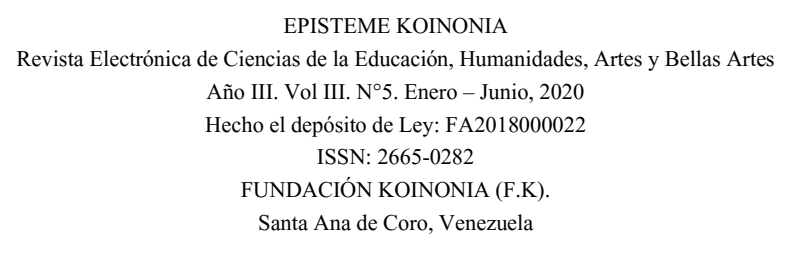

Raysa Chiquinquirá Chirino-García; Janeth Hernández-Corona

de las tecnologías subyacentes de computación cognitiva -como el aprendizaje profundo (deep learning), en aprendizaje automático (machine learning) y las redes neuronales-, son conocidos lo que la convierte como piedra angular para el aprendizaje flexible y colaborativo, constituyéndose en una herramienta importante para la toma de decisiones.

De acuerdo con las teorías planteadas, se puede advertir que el aprendizaje electrónico móvil ofrece métodos modernos de apoyo al proceso enseñanza-aprendizaje mediante el uso de dispositivos móviles; tales como: Tablet-PC, Personal digital assistant (PDA), MP3, los teléfonos inteligentes (Smartphone), los teléfonos móviles y los ordenadores portátiles, entre otros. En síntesis, se puede acotar que, el aprendizaje electrónico móvil se presenta como un elemento ligado invariablemente a todo dispositivo de mano que tenga alguna forma de conectividad inalámbrica convirtiéndose en una herramienta habitual en la vida de mucha gente, donde éste se está convirtiendo en solución a los problemas que enfrenta el sector educativo.

En este sentido, constituye un apoyo las partes intervinientes en el proceso enseñanzaaprendizaje puesto que en su conducto la instrucción puede realizarse en cualquier lugar, así como en todo momento. En consecuencia, el uso de los dispositivos móviles, así como las nuevas tecnologías coadyuvan a la instrucción formal e informal puesto que posee un gran potencial para transformar los rendimientos educativos y la capacitación. De allí, la importancia que reviste para el presente estudio.

\section{Ventajas y desventajas del aprendizaje electrónico móvil en la educación superior} En la práctica educativa, se han gestado una congerie de cambios que se orientan hacia el uso cada vez más recurrente del internet aunado al uso de dispositivos de mano con conectividad inalámbrica cuyas características lo convierten en aliados de los estudiantes así como de las instituciones educativas puesto que éstas últimas propician la implementación de estrategias que posibiliten la creación en ellos del espíritu de 


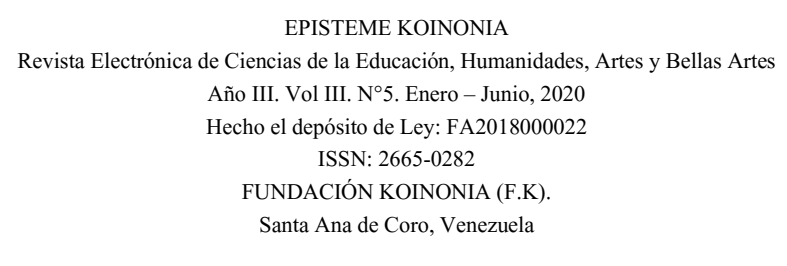

Raysa Chiquinquirá Chirino-García; Janeth Hernández-Corona

cooperación e innovación, mentes críticas, destrezas tecnológicas de manera tal que se fortalezca y fomente un aprendizaje colaborativo.

Dentro de este contexto, Hernández \& Morales (2010), señalan varias utilidades e inconvenientes en la aplicación del M-Learning. En este sentido, dentro de las bondades en la aplicación de la metodología indicada, se tiene:

- Aumento del tiempo útil y de la disponibilidad geográfica.

- El tiempo para desarrollar actividades de enseñanza y aprendizaje puede ser incrementado.

- Mayor autonomía.

- El estudiante puede personalizar el equipo móvil de acuerdo con sus requerimientos.

- Contenido adaptado a la ubicación física.

- El dispositivo móvil puede mostrar la información de lo que está más cerca de él en cuanto al tiempo del que desea obtener información, los intereses específicos o el lugar hacia donde se dirige.

- Necesidad de conexión.

- El acceso inalámbrico puede ser convenientemente escalado para diferentes usuarios.

- Acceso inmediato a datos y avisos.

- Los usuarios pueden acceder en forma inmediata a ciertos datos que debe entregar en un corto tiempo, entre otros.

- Autenticación segura.

- Los dispositivos móviles pueden proveer un mecanismo conveniente para identificar el usuario y algunos métodos flexibles de pago.

- Alta Personalización.

- En un ambiente ampliamente abierto hasta el punto que el usuario sea un desarrollador de contenidos y servicios útiles para todos los usuarios.

- Alta expansión de la tecnología.

Adicionalmente a lo comentado anteriormente, el hecho de que la extensión de la tecnología móvil sea cada vez mayor además que un porcentaje alto de la población posea uno o varios terminales móviles favorece en gran medida el aprendizaje mediante el uso de ese tipo de dispositivos: 
- Pequeña curva de aprendizaje.

- Reduce los periodos de formación.

- Mayor libertad y flexibilidad de aprendizaje.

- Ofrecen la libertad de acceder a las tecnologías de la información cuando y donde el usuario lo necesite, facilitando la posibilidad de implementar innovadores modos de dar clase y aprender.

Continúan resaltando Hernández \& Morales (2010), sobre algunos de los inconvenientes en uso del M-Learning, dentro de los cuales se tiene:

- Resistencia al cambio por parte de algunos estudiantes o docentes.

- Dificultad de adaptación a los dispositivos móviles.

- Carencia de periféricos para el dispositivo móvil que limitan al usuario a modelos de aprendizaje básico.

- Con excepción de los Tablet PC y los portátiles, presentan problemas asociados a la usabilidad, debido a las pantallas pequeñas.

- La navegación suele ser limitada.

- Los costos de acceso a la red son altos.

- El trabajo colaborativo es menor.

- La Necesidad de conexión inalámbrica que se puede ver afectada por la ubicación física del usuario.

Sobre la base de las ideas planteadas anteriormente, las investigadoras se evidencian las ventajas del M-Learning; puesto que, se presentan como un nuevo paradigma para el aprendizaje colaborativo. De manera que, los entornos constructivistas tienen grandes beneficios puesto que el uso de esta estrategia le permitirá al estudiante realizar las actividades a su ritmo ya que cada estudiante podrá discernir sobre la importancia de hacer aportes que redundaran en beneficios para él, así como para el grupo o comunidad académica.

En resumen, se daría cumplimiento a una de las metas preestablecidas en la educación contemporánea, puesto que, al prestarle atención a un número significativo de estudiantes, se le estaría dando respuesta a una demanda no satisfecha además de incrementar y facilitar posibilidades de impartir docencia a aquellas personas que por 
diversas razones están imposibilitadas de acudir a ambientes de educación formal tradicional.

\section{M-Learning en la educación superior}

Desde hace varios años, el modelo educativo basado en el uso de dispositivos móviles se ha sustentado en diferentes tipos de aprendizaje, el cual puede variar en función de las características en cada uno de ellos. Sin embargo, no son excluyentes además se pueden mezclar y alcanzar patrones mixtos que combinen lo mejor de cada tipo para crear uno más integral.

Por su parte Hernández (2010), comenta que el uso en el aula del M-Learning, facilita la comprensión de los conocimientos; incluye multimedia y está centrado en el entorno, propicia la atención a la diversidad además que favorece el aprendizaje centrado en el alumno y en el contexto. Por otro lado, fomenta la interacción y comunicación además de permitir el intercambio de datos entre los miembros de la comunidad educativa (alumnos y profesor), proporciona la publicación directa de contenidos y comentarios, entre otros.

Del mismo modo, enfatiza Hernández (2010) que el M-Learning es un escenario virtual donde la construcción del conocimiento constituye un proceso activo puesto que es desde la experiencia de los estudiantes y los tutores en línea que se genera un desarrollo dinámico de carácter social, pero con pertinencia académica. Por consiguiente, se deben evaluar las estrategias de seguimiento o monitoreo, así como para mantener el control de las aulas virtuales la metodología M-Learning representa una herramienta excelente que proporciona registros que coadyuvan a medir el desempeño de los alumnos. En este sentido, con esa aplicación, la institución de educación busca a lograr el mejoramiento continuo. En esta instancia, el binomio académico y tecnológico en esta metodología constituye un proceso activo donde las 
partes intervinientes (institución de educación superior, tutor y alumnos), desempeñan un rol dinámico y reflexivo.

En otro orden de ideas, Herrera (2012) advierte que la aplicación del M-Learning, resulta muy efectivo para los aprendizajes basados en la resolución de problemas, para la adquisición de habilidades, en el trabajo de campo, en estudios exploratorios, entre otros. Desde otra perspectiva, Marqués (2013) sostiene que esta metodología de aprendizaje móvil admite la evaluación formativa y sumativa además de aumentar la motivación del estudiante. En síntesis, el aprendizaje electrónico móvil representa una ventana tecnológica para la modalidad educativa, la cual coadyuva la construcción del conocimiento, la resolución de problemas de aprendizaje, así como el incremento de una congerie de actitudes y aptitudes, las cuales facilitan el acceso de manera autónoma y ubicua gracias a la utilización de los dispositivos móviles portables.

\section{Herramientas de aprendizaje social (Social Learning tools)}

Conocidas también como "Social learning tools", son consideradas como herramientas de aprendizaje social sean han convertido en aquellos recursos utilizados en el proceso enseñanza aprendizaje con propósitos andragógicos y pedagógicos, manejando un software social o medios de comunicación social con el propósito de facilitar el aprendizaje en la interacción entre personas y sistemas.

Por otro lado, I Vila (2012) sostiene que, en la actualidad, los servicios de las redes digitales permiten de forma sencilla hacer públicos los miembros que forman parte de ellas, sus conocimientos y sus colaboraciones desde casi cualquier lugar en cualquier momento, para intercambiar información, ideas y experiencia velando por su crecimiento personal y profesional, permitiendo crear sistemas de comunicación significativa y de cohesión social.

Para el autor, existen dos opciones para que las redes entren en el proceso de enseñanza y con qué estrategias, una opción es dejarlas fuera del muro de las aulas sin 


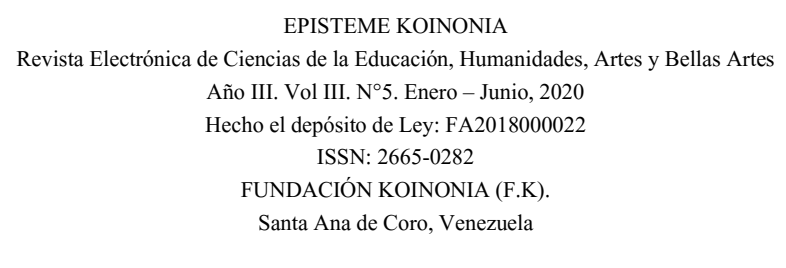

Raysa Chiquinquirá Chirino-García; Janeth Hernández-Corona

aprovechar su potencial educativo del uso didáctico de los móviles. La otra opción es aprovechar que los alumnos ya forman parte de ellas, las interacciones se registran, facilitando la evaluación de los procesos y la detección de situaciones conflictivas fuera de la red.

Por otra parte, González (2014) expresa que, las redes sociales y los edublogs como herramientas de aprendizaje colaborativo han mostrado nuevos entornos virtuales para el aprendizaje mediante el uso de tecnologías activas, al internet como el gran fenómeno sociocultural y el desarrollo de la web, conjuntamente con una diversidad de herramientas, han abierto a una multitud de posibilidades para distintos aspectos como la formación, el uso de modelos constructivistas en modelos de enseñanza aprendizaje, procesos de innovación y mejora, así como en el aprendizaje colaborativo.

En ese orden de ideas, Montero \& Gewerc (2014) han determinado en sus estudios que el uso de una red social se originó de la necesidad de encontrar dispositivos que combinen los aspectos sociales y colaborativos del aprendizaje con la construcción que cada sujeto realiza en los entornos personales con los que aprende. Consideran que la construcción social del concepto de juventud y de las competencias que los jóvenes necesitan para adaptarse a la nueva economía basada en el conocimiento es utilizado también como reclamo para provocar transformaciones educativas.

Según la opinión de los autores, con el argumento de partir de las necesidades y expectativas construidas en su proceso de socialización con las tecnologías, se debe plantear un cambio educativo profundo, tanto en el curriculum como en las instituciones y en los roles que debe asumir el profesorado. Las nuevas tecnologías se han convertido en un nuevo escenario educativo proyectando las posibilidades que ofrece la web y estimulando a que el estudiante sea activo en el proceso de aprendizaje conectando con una comunidad virtual de aprendizaje (UNED 2018).

En esa perspectiva, Visa B, (2014) considera que el aprendizaje colaborativo y el uso de la tecnología son dos tendencias que están en vigor en todas las etapas educativas 
del individuo, es decir, desde primaria hasta la etapa universitaria, encontrando una multitud de aplicaciones, plataformas virtuales que se están implementando de manera espectacular, mejorando a ritmo vertiginoso para poder la adquisición de contenidos inherentes a una materia, a la par fomentar la interacción entre docentes, alumnos además de la colaboración entre los alumnos (UNED, 2018).

En otro orden de ideas, Cerezo (2008) citado por Martínez (2014) menciona que el interés por las redes sociales no radica tanto en los contenidos como en su capacidad para establecer conexiones y agregar valor, ya que es la importancia creciente en los nodos interconectados frente al conocimiento individualizado. Para Martínez, (2014) el termino e-learning se utiliza para referirse al aprendizaje que se comparte y se construye con los propios alumnos que mezclan y reorganizan la distribución de un contenido, adquiriendo competencias digitales y la consecución de objetivos formativos. Para la autora, cuando se centraliza el e-learning en un sistema de gestión del aprendizaje o plataformas, puede abrirse un ecosistema digital adoptando características de la conectividad, estableciendo conexiones con sistemas externos, vía mashups, que es el que encierra el proceso creativo de recombinar la información existente y crear nueva información con esos datos, la convergencia anima a los usuarios a establecer conexiones entre contenidos dispersos de los medios, creando y descubriendo nuevas formas de información que se visualizan en formatos y espacios diferentes (González Isasi \& Medina Morales, 2018).

Continua Martínez (2014) manifestando que la enseñanza al aprendizaje postelearning, reemplaza la simple transmisión de la información de la enseñanza por un modelo abierto de conocimiento basado en redes interrelacionadas, abierto a nuevos itinerarios de formación y a todos los públicos, siendo activos en el proceso, teniendo en cuenta los estilos cognitivos de los sujetos y la variedad de dispositivos que dan acceso a un conocimiento multisensorial verbal, escrito, visual y sonoro, ajustándose a 


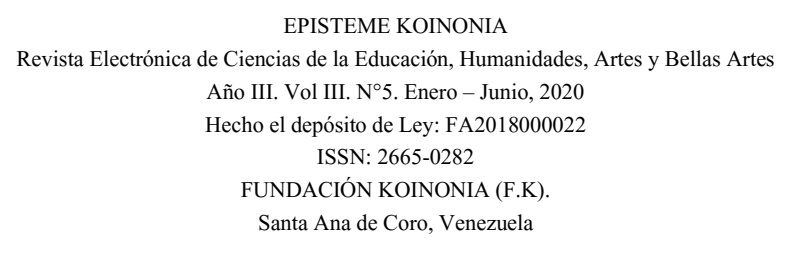

Raysa Chiquinquirá Chirino-García; Janeth Hernández-Corona

una nueva pedagogía adaptada a una comunicación móvil, flexible, no lineal, adaptada a los intereses y necesidades de los aprendices.

Para Martínez (2014) el potencial mashups aplicados al contexto educativo radica en la forma en que posibilitan llegar a nuevas conclusiones o discernir nuevas relaciones mediante la unión y combinación de grandes cantidades de datos, procedentes de distintas fuentes, de una manera manejable, por lo cual existen herramientas que proporcionan un entorno inmersivo que pueden integrarse desde los mundos virtuales de manera que el usuario en un mismo espacio pueda acceder a una exposición, un video o un wiki, tales como: There, Active Worlds, Olive y Second Life, permiten la interacción de aplicaciones y recursos.

\section{REFLEXIONES DE CIERRE}

En la sociedad actual, la realidad educativa se vincula estrechamente con los avances e innovaciones tecnológicas pues M-Learning o aprendizaje electrónico y la promoción del aprendizaje electrónico móvil, ambos son posibles mediante el uso de plataformas informáticas, lo que representa una ventaja; puesto que, el saber en la práctica social debe ser considerado por los diferentes sectores de gran relevancia. De allí que, el desafío actual para las partes involucradas en este tema, demanda la satisfacción de los requerimientos empresariales aunado a la formación de un ser humano con una preparación superior que contemple la práctica con medios telemáticos, así como la utilización de plataformas tecnológicas para la transmisión y generación del conocimiento.

Volviendo la mirada a lo anterior, las investigadoras asienten que el uso de la metodología M-Learning o aprendizaje móvil, coadyuva mancomunadamente al binomio sociedad-instituciones de educación; puesto que, aportan soluciones a los problemas de la sociedad globalizada; del mismo modo; asimismo, no solo atiende y da respuesta a la problemática del espacio físico sino que puede ser utilizada con éxito ante cualquier crisis global o contingencias, además, brinda beneficios de optimización del tiempo, 


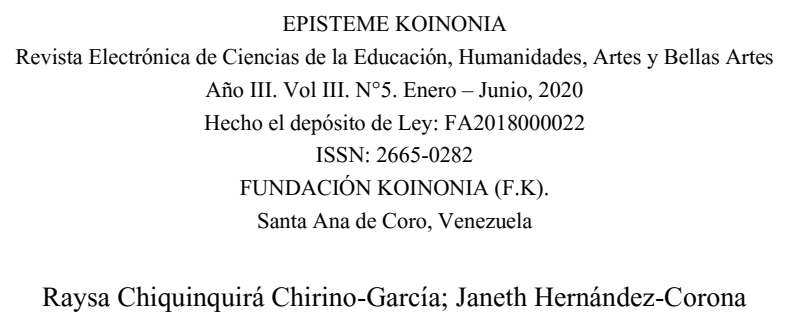

economía en espacios físicos, reducción de costos que se originan producto de las distancias y desplazamientos frecuentes de las partes intervinientes. De ahí que, tanto el aprendizaje electrónico como el aprendizaje electrónico móvil, ofrecen un valioso apoyo en el sector educativo, entre otras razones, es una moderna y efectiva vía para la generación y transmisión de conocimientos que favorecen la evolución de la actual sociedad de la información y comunicación.

Finalmente, las investigadoras advierten que deberá quedar claro que el M-Learning de ninguna manera representa un sustituto de la formación tradicional pues ambas pueden coexistir perfectamente en función de las necesidades, además dado el vertiginoso auge de las nuevas tecnologías, se puede afirmar que los estudiantes conocen bien los diferentes medios informáticos pero los docentes (profesores, tutores y facilitadores, entre otros) son los que manejan la tecnología cognitiva, además que, su misión es enseñarles cómo optimizar y usar dichos dispositivos o instrumentos móviles pero con fines académicos; de ahí el reto al que deben enfrentarse.

\section{FINANCIAMIENTO}

No monetario

\section{AGRADECIMIENTOS}

Especial agradecimiento al personal docente de la Universidad Privada Dr. Rafael Belloso Chacín por su estímulo en el desarrollo de esta investigación.

\section{REFERENCIAS}

Aldana Zavala, J. J. (2019). La competencia epistemológica en el investigador social universitario venezolano. [Epistemological competence in the Venezuelan university social researcher]. Praxis, 15(1), 103-115. https://doi.org/10.21676/23897856.3091 


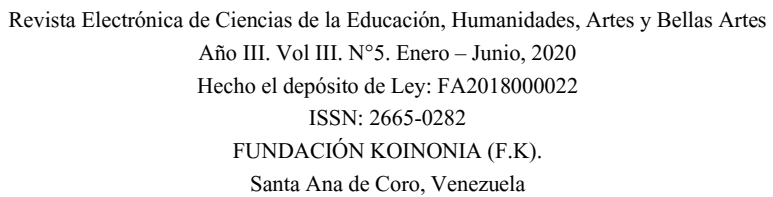

Raysa Chiquinquirá Chirino-García; Janeth Hernández-Corona

Anderson, C., \& Andara, Z. (2017). Entorno virtual de apoyo al aprendizaje para el curso peritaje laboral judicial ofertado por el departamento de postgrado de la UPTAG. [Virtual learning support environment for the judicial labor appraisal course offered by the UPTAG graduate department]. IUSTITIA SOCIALIS, 2(2), 45-61. Recuperado de https://n9.cl/st3j

Arias, F (2012). El proyecto de Investigación: Introducción a la investigación científica. [The Research project: Introduction to scientific research]. 6ta. Edición. Editorial Episteme.

Brazuelo Grund, F. \& Gallego Gil, D. (2011). Mobile Learning: los dispositivos móviles como recurso educativo. [Mobile Learning: mobile devices as an educational resource]. Sevilla: Editorial MAD Eduforma. ISBN: 978-84- 676-5706-7

Cabal, C, Martínez, F y Molina, V. (2010). Sistemas de recomendación en el Comercio Electrónico y la E-educación. [Recommendation systems in Electronic Commerce and E-education]. Universidad libre de Bogotá. Criterio Libre - Vol. 8 • No. 12 • Bogotá, Colombia - Enero - junio 2010 - Pp. 161-182.

Cavaller, V. (2016). La tecnología cognitiva. [Cognitive technology]. Recuperado de https://n9.cl/6yegk

González, C (2014). Metodologías de aprendizajes colaborativos a través de las tecnologías. Redes sociales y los edublogs como herramientas de aprendizaje colaborativo. [Collaborative learning methodologies through technologies. Social networks and edublogs as collaborative learning tools]. Salamanca, España. Ediciones Universidad de Salamanca.

González Isasi, R., \& Medina Morales, G. (2018). Uso de dispositivos móviles como herramientas para aprender Píxel-Bit. [Using mobile devices as tools to learn Pixel-Bit]. Revista de Medios y Educación, 0(52), 217-227. http://dx.doi.org/10.12795/pixelbit.2018.i52.15

Hernández, R, \& Morales, M. (2010). Dispositivos móviles en educación. [Mobile devices in education]. Revista América Learning \& Media. Recuperado de https://n9.cl/ae16e

Hernández, T (2010). M-learning como Estrategia de Diversificación en Ambientes Colaborativos. [M-learning as a Diversification Strategy in Collaborative Environments]. Universidad Dr. Rafael Belloso Chacín. Maracaibo. Venezuela. 


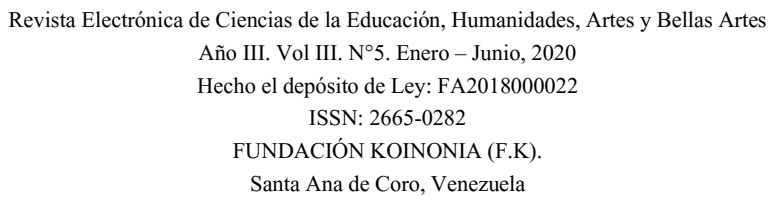

Raysa Chiquinquirá Chirino-García; Janeth Hernández-Corona

Herrera, B. (2012). El móvil en la educación: Un nuevo paradigma. Ventajas y desventajas de su uso. [The mobile in education: A new paradigm. Advantages and disadvantages of its use]. Recuperado de https://n9.cl/hxw5m

I Vila, J. (2012). Aprendizaje social y personalizado: conectarse para aprender. Colección Educación Sociedad y Red. [Social and personalized learning: connect to learn. Collection Education Society and Network]. Recuperado de https://n9.cl/yste

Kinshuk, D (2009). Adaptive Mobile Learning Techonologies. Athabasca University. Recuperado de https://n9.cl/aulz

Marqués, P (2013). Claves para mejorar los aprendizajes integrando las tecnologías móviles en las clases" en Tecnología móvil e innovación en el aula. Nuevos retos y realidades educativas. [Keys to improve learning by integrating mobile technologies in classes "in Mobile Technology and innovation in the classroom. New challenges and educational realities].Jornadas Internacionales (2013) Universidad de La Rioja. Recuperado de https://n9.cl/aulz

Martínez, R (2014). Sloodle: Conexión de entornos de aprendizajes. [Sloodle: Connecting learning environments]. Barcelona, España. Editorial UOC.

Mero Chávez, E., Fernando Pazmiño, M., \& San Andrés, E. (2019). El flipped classroom como herramienta innovadora para el desarrollo del aprendizaje significativo. [The flipped classroom as an innovative tool for the development of meaningful learning]. Cienciamatria, 6(10), 646-661. https://doi.org/10.35381/cm.v6i10.281

Montero, L, \& Gewerc, A. (2014). Educación, medios digitales y cultura de la participación. De las redes para el ocio de las redes sociales para la academia. Un estudio de caso. [Education, digital media and culture of participation. From networks for leisure to social networks for academia. A case study]. Barcelona España. Editorial UOC.

Palella, S y Martins, P. (2012). Metodología de la investigación cualitativa. [Qualitative research methodology]. 3era edición. FEDUPEL. Caracas-Venezuela.

Peche Cruz, H., \& Giraldo Supo, V. (2019). El Aprendizaje Flip Learning centrado en el estudiante como generador de calidad educativa. [Student-centered Flip Learning as a generator of educational quality]. Revista Arbitrada Interdisciplinaria Koinonía, 4(8), 427-450. http://dx.doi.org/10.35381/r.k.v4i8.293 


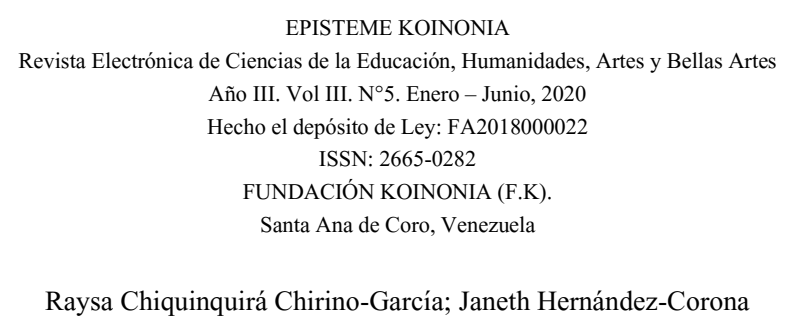

Perozo Martín, R., \& Chirinos Martínez, A. (2019). Incidencias de la Tecnología web 2.0 en el contexto de la gobernanza y la gobernabilidad. [Incidences of Web 2.0 Technology in the context of governance and governability]. IUSTITIA SOCIALIS, 4(6), 90-116. http://dx.doi.org/10.35381/racji.v4i6.291

UNED (2018). Los recursos móviles en educación. De la pizarra tradicional a la tableta digital. [Mobile resources in education. From the traditional blackboard to the digital tablet]. Recuperado de https://n9.cl/287xv

UNESCO (2019). Aprendizaje móvil. [Mobile learning]. Recuperado de https://n9.cl/okij

Velazco Flórez, S. Y., Abuchar Porras, A., Castilla, I., \& Rivera, K. (2017). E-Learning: Rompiendo fronteras. [E-Learning: Breaking boundaries]. Redes De Ingeniería, 91-100. https://doi.org/10.14483/2248762X.12480

Visa B, M. (2014) El EEES como marco de desarrollo de las nuevas herramientas docentes. [The EHEA as a framework for the development of new teaching tools]. Madrid, España. Editorial ACCI.

Xiao, B. \& Benbasat, I. (2007). E-commerce product recommendation Agents: Use, Characteristics, and Impact, in: MIS Quaterly, Vol. 31, No. 1, pp. 137-209.

(C)2020 por el autor. Este artículo es de acceso abierto y distribuido según los términos y condiciones de la licencia Creative Commons Atribución-NoComercial-Compartirlgual 4.0 Internacional (CC BY-NC-SA 4.0) (https://creativecommons.org/licenses/by-nc-sa/4.0/). 